\title{
Enhancing thj production from top-Higgs FCNC couplings
}

\section{Lei $\mathbf{W u}$}

ARC Centre of Excellence for Particle Physics at the Terascale, School of Physics, The University of Sydney,

Sydney, New South Wales, 2006 Australia

E-mail: leiwu@physics.usyd.edu.au

ABSTRACT: In this paper, we study the single top and Higgs associated production $p p \rightarrow$ th $j$ in the presence of top-Higgs FCNC couplings at the LHC. Under the current constraints, we find that the full cross section of $p p \rightarrow t h j$ can be sizably enhanced in comparison with the SM predictions at 8 and $14 \mathrm{TeV}$ LHC. We further explore the observability of top-Higgs FCNC couplings through $p p \rightarrow t\left(\rightarrow b \ell^{+} \nu_{\ell}\right) h(\rightarrow \gamma \gamma) j$ and find that the branching ratios $B r(t \rightarrow q h), B r(t \rightarrow u h)$ and $B r(t \rightarrow c h)$ can be respectively probed to $0.12 \%, 0.23 \%$ and $0.26 \%$ at $3 \sigma$ sensitivity at $14 \mathrm{TeV}$ LHC with $\mathcal{L}=3000 \mathrm{fb}^{-1}$.

KEywords: Rare Decays, Beyond Standard Model

ARXIV EPRINT: 1407.6113 


\section{Contents}

1 Introduction 1

2 Top-Higgs FCNC interactions 3

3 Numerical calculations and discussions 3

4 Conclusion $\quad 9$

\section{Introduction}

The discovery of the Higgs boson at the LHC is a great triumph of the Standard Model (SM) and marks a new era in the particle physics [1,2]. Given the large uncertainties of the current Higgs data, there remains a plenty of room for new physics in Higgs sector [3, 4]. ${ }^{1}$ So the precise measurement of the Higgs boson's properties will be a dominant task at the LHC in the next decades.

Concerning the probe of new physics through the Higgs boson, the Yukawa couplings can play the important role since they are sensitive to new flavor dynamics beyond the SM. In particular, top quark, as the heaviest SM fermion, owns the strongest Yukawa coupling and has the preference to reveal the new interactions at the electroweak scale [5$14] .{ }^{2}$ One of the interesting things is that the top quark is just heavier than the observed Higgs boson, which makes the top quark flavor changing neutral current (FCNC) processes $t \rightarrow h q(q=u, c)$ be accessible in kinematics. In the SM, these top quark FCNC transitions are extremely suppressed by the G.I.M. mechanism [15-18]. But they can be greatly enhanced by the extended flavor structures in many new physics models, for example the minimal supersymmetric model (MSSM) with/without R-parity [19-32], two-Higgsdoublet model (2HDM) type-III [33-45], and the other miscellaneous models [46-60]. So the study of top-Higgs FCNC interactions is a common interest of the theory and experiment communities [61-67]. ${ }^{3}$ However, up to now, the null results of the searches for $t \rightarrow q h$ at the LHC give the strong limits on the top-Higgs FCNC couplings. Among them, the most stringent constraint $\mathrm{Br}(t \rightarrow h c)<0.56 \%$ at $95 \%$ C.L. was reported by the CMS collaboration from a combination of the multilepton channel and the diphoton plus lepton channel [67]. Except for the widely studied $t \rightarrow q h$ decays, the importance of the single top+Higgs production $p p \rightarrow$ th in probing the top-Higgs FCNC couplings has been also emphasized in the recent theoretical studies [68-73].

\footnotetext{
${ }^{1}$ The recent reviews, see examples.

${ }^{2}$ For top quark reviews, see, e.g.

${ }^{3}$ For reviews on top FCNC processes in new physics models, see, e.g.
} 

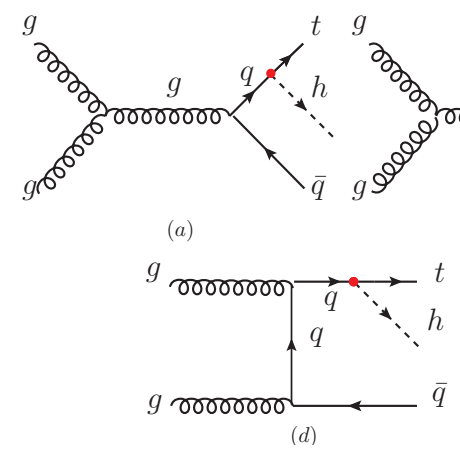

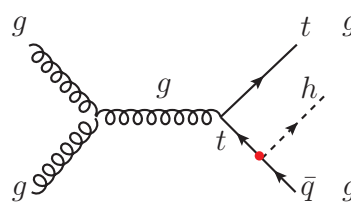

$(b)$

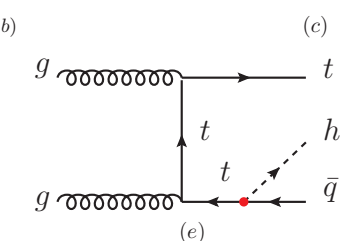

Figure 1. Example Feynman diagrams for the partonic process $g g \rightarrow t h \bar{q}$ at the LHC through flavor violating top-Higgs interactions in eq. (2.1)(marked with red dots). Here $q=u, c$.

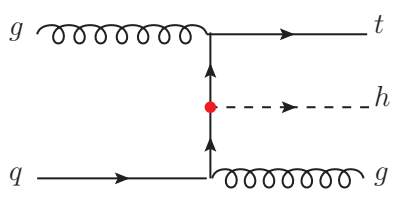

$(a)$

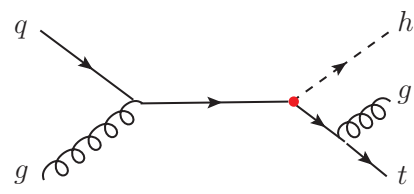

$(b)$

Figure 2. Example Feynman diagrams for the partonic process $q g \rightarrow$ thg at the LHC through flavor violating top-Higgs interactions in eq. (2.1) (marked with red dots). Here $q=u, c$.

In this paper, we investigate the top-Higgs FCNC interactions through $p p \rightarrow t h j$ with the sequent decays $t \rightarrow b \ell^{+} \nu$ and $h \rightarrow \gamma \gamma$ at the LHC. In the SM, the process $p p \rightarrow t h j$ can only be induced by the weak charged current interaction and has a relative small cross section, which is about 18 (88) fb at 8 (14) TeV LHC. However, such a process is found to be very sensitive to modifications of the Higgs couplings [74-81]. In particular, the top-Higgs FCNC couplings $t q h(q=u, c)$ can sizably enhance thj cross section due to the contributions from the strong interaction processes. To be specific, there are mainly three new kinds of processes that can contribute to the production of thj at the LHC: (1) gluon fusion $g g \rightarrow t h j$, it is the dominant contribution, as shown in figure 1, where $h j$ can be produced not only by an on-shell top quark but also by an off-shell top quark via the new flavor changing couplings $t q h$; (2) $q g$ fusion $q g \rightarrow t h j$, as shown in figure 2, which is the sub-leading contribution. However, such a process is affected by the initial Parton Distributions Functions (PDFs). So one can use this feature to disentangle the FCNC couplings of the top quark with light quarks; (3) $q \bar{q}$ annihilation $q \bar{q} \rightarrow t h j$, which is similar to the $s$-channel of the process $g g \rightarrow t h j$ but with $q \bar{q}$ instead of $g g$ in the initial states. The contribution of this process is relatively smaller than (1) and (2) because of the suppression of color factor and PDFs; based on the above considerations, it is worthwhile to perform a complete calculation of $p p \rightarrow t h j$ in the presence of the top-Higgs FCNC couplings by including the contributions (1)-(3), and explore its sensitivity to probe the top-Higgs FCNC couplings at the LHC.

This paper is arranged as follows. In section 2, we set up the notations and briefly describe the top-Higgs FCNC interactions. In section 3, we discuss the observability of 
the top-Higgs FCNC couplings through the process $p p \rightarrow$ th $j$ at $14 \mathrm{TeV}$ LHC. Finally, a summary is given in section 4 .

\section{Top-Higgs FCNC interactions}

A general effective Lagrangian describing the top-Higgs FCNC interaction can be written as

$$
-\mathcal{L}_{t q h}=\kappa_{t q h}^{L} \bar{t}_{L} q_{R} h+\kappa_{t q h}^{R} \bar{t}_{R} q_{L} h+h . c .
$$

where $h$ is the SM Higgs boson, and the real parameter $\kappa_{t q h}^{L, R}$ denote the left-handed and right-handed FCNC couplings of the Higgs boson to the light up-type quarks $q=u, c$. We plot example Feynman diagrams in figure 1 and figure 2 for the partonic process $g g \rightarrow t h \bar{q}$ and $q g \rightarrow t h g$, respectively. Some diagrams of the process $u \bar{u} / d \bar{d} \rightarrow t h \bar{q}$ can be obtained by replacing the initial gluons with $u \bar{u} / d \bar{d}$ in the $s$-channel in figure 1. By neglecting the light quark masses and assuming the dominant top decay width $t \rightarrow b W$, the Leading Order (LO) branching ratio of $t \rightarrow q h$ can be approximately given by,

$$
B r(t \rightarrow q h)=\frac{\kappa_{t q h}^{L}{ }^{2}+\kappa_{t q h}^{R}{ }^{2}}{2 \sqrt{2} m_{t}^{2} G_{\mathrm{F}}} \frac{\left(1-x_{h}^{2}\right)^{2}}{\left(1-x_{W}^{2}\right)^{2}\left(1+2 x_{W}^{2}\right)} .
$$

where $G_{\mathrm{F}}$ is the Fermi constant, $x_{W}=m_{W} / m_{t}$ and $x_{h}=m_{h} / m_{t}$. The NLO QCD correction to $\mathrm{Br}(t \rightarrow q h)$ is estimated as $10 \%$ according to the results of high order corrections to $t \rightarrow b W$ [82] and $t \rightarrow c h$ [83-85]. In some specific models, the left-handed coupling $\kappa_{t q h}^{L}$ is not expected to be large because its relation with the CKM mixing parameter. Also, $\sqrt{{\kappa_{t q h}^{L}}^{2}+\kappa_{t q h}^{R}{ }^{2}}$ can be constrained by the low energy observables, such as $B^{0}-\overline{B^{0}}$ mixing $[45,86]$. However, we do not consider these indirect constraints in our study since they are model-dependent and their relevance strongly depends on the assumptions made for the generation of the quark flavor structures [87-89]. On the other hand, the CMS collaboration reported a model-independent bound $\sqrt{{\kappa_{t q h}^{L}}^{2}+{\kappa_{t q h}^{R}}^{2}}<0.14$ at 95\% C.L. from the combined result of multilepton and diphoton in $t \bar{t}$ production [67], which indicates $\left|\kappa_{t q h}^{L, R}\right|$ should be less than 0.14. In our work, we assume $\kappa_{t q h}^{L}=\kappa_{t q h}^{R}=\kappa_{t q h}$ and require $\kappa_{t q h} \leq 0.1$ to satisfy the direct constraint from the CMS result.

\section{Numerical calculations and discussions}

We implement the top-Higgs FCNC interactions by using the package FeynRules [90] and calcualte the LO cross section of $p p \rightarrow$ thj with MadGraph5 [91]. We use CTEQ6L as the parton distribution function (PDF) [92] and set the renormalization scale $\mu_{R}$ and factorization scale $\mu_{F}$ to be $\mu_{R}=\mu_{F}=\left(m_{t}+m_{h}\right) / 2$. The SM input parameters are taken as follows [93]:

$$
\begin{aligned}
& m_{t}=173.07 \mathrm{GeV}, \quad m_{Z}=91.1876 \mathrm{GeV}, \quad \alpha\left(m_{Z}\right)=1 / 127.9, \\
& \sin ^{2} \theta_{W}=0.231, \quad m_{h}=125 \mathrm{GeV}, \quad \alpha_{s}\left(m_{Z}\right)=0.1185 .
\end{aligned}
$$



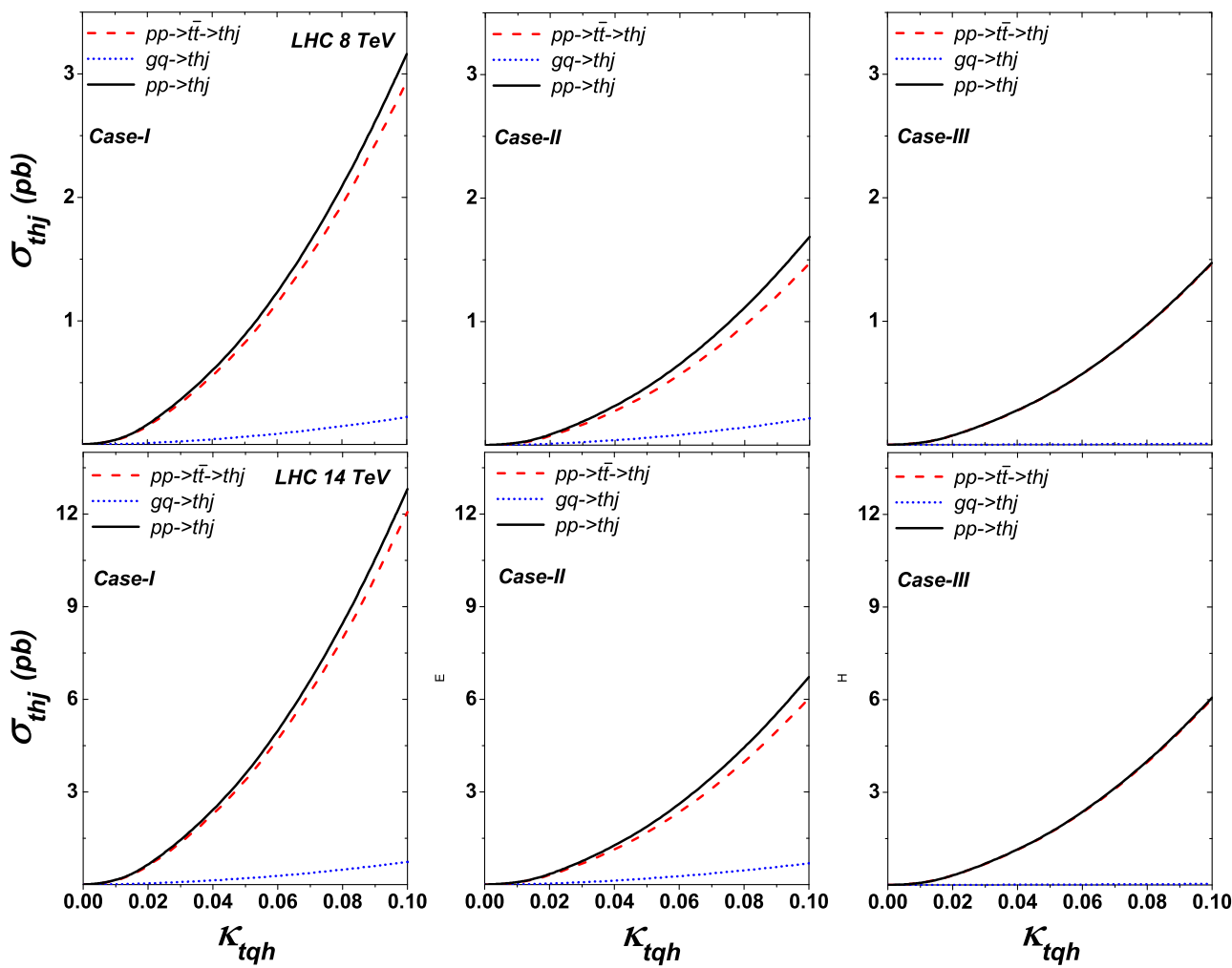

Figure 3. The dependence of the cross sections $\sigma_{t h j}$ at 8 and $14 \mathrm{TeV}$ LHC on the top-Higgs FCNC couplings $\kappa_{t q h}$ for case (I)-(III). The conjugate processes have been included in the calculations.

In figure 3, we show the dependence of the cross sections $\sigma_{t h j}$ on the top-Higgs FCNC couplings $\kappa_{t q h}$ at 8 and $14 \mathrm{TeV}$ LHC respectively for three different cases: (I) $\kappa_{t q h}=\kappa_{t u h}=$ $\kappa_{t c h}$, (II) $\kappa_{t q h}=\kappa_{t u h}, \kappa_{t c h}=0$ and (III) $\kappa_{t q h}=\kappa_{t c h}, \kappa_{t u h}=0$. For the three cases, the main contribution to $p p \rightarrow t h j$ is from the resonant production $p p \rightarrow t \bar{t} \rightarrow t h j$. The non-resonant contributions are dominated by the process $g q \rightarrow t h j$. To be specific, we can have the following observations:

- Case- $(I)$ : when $\kappa_{t q h}=0.1$, the total cross section of $p p \rightarrow$ thj at 8 and $14 \mathrm{TeV}$ LHC can be respectively enhanced up to nearly 176 and 145 times the SM predictions [76]. For the smaller values of $\kappa_{t q h}$, the cross section will decrease and become comparable with the SM prediction when $\kappa_{t q h} \sim 0.008$. Here it should be mentioned that although the CMS collaboration has performed a search for $t h j$ event at $\sqrt{s}=8 \mathrm{TeV}$ and given a $95 \%$ upper limit on the $t h j$ cross section $\sigma_{t h j}<2.24 \mathrm{pb}$, this bound is not suitable for our case because a forward jet with $|\eta|>1.0$ is required in the experimental analysis. We can also see that the full cross section of $p p \rightarrow t h j$ is 1.08 (1.06) times larger than the one of $p p \rightarrow t \bar{t} \rightarrow t h j$ at 8 (14) TeV LHC due to the contributions of the non-resonant productions of $h j$.

- Case- $(I I)$ and (III): for the same values of $\kappa_{t u h}$ and $\kappa_{t c h}$, the cross section of $p p \rightarrow t h j$ in case- $(I I)$ is much larger than that in case- $(I I I)$, since the up-quark has the larger 
PDF than the charm-quark. This feature allows us to separately probe the couplings between $\kappa_{t u h}$ and $\kappa_{t c h}$ at the LHC. So, in general, for a given collider energy and luminosity, we can expect the sensitivity to the coupling $\kappa_{t u h}$ will be better than $\kappa_{t c h}$. It should be also mentioned that the dominant contribution to $p p \rightarrow t h j$ in case-(II) and case- $(I I I)$ still come from $g g$-fusion process. The main difference between case$(I I)$ and case- $(I I I)$ lies in the contribution of $q g \rightarrow t h j$ process. This makes the complete cross section of $t h j$ almost same as that of $t \bar{t} \rightarrow t h j$ in case-(III) because of the small portion of $c$ quark in the proton. To be specific, when $\sqrt{s}=8(14) \mathrm{TeV}$ and $\kappa_{t q h}=0.1, \sigma_{p p \rightarrow t h j}$ is about 1.16(1.12) and 1.006(1.005) times larger than $\sigma_{p p \rightarrow t \bar{t} \rightarrow t h j}$ in case- $(I I)$ and $-(I I I)$, respectively.

- Case- $(I)$ and $(I I)$ : we also find that the impact of $q g \rightarrow t h j$ on increasing the cross section of thj production in case- $(I)$ is smaller than that in case- $(I I)$. The reason is that the main production mode $g g \rightarrow t h j$ in case- $(I)$ includes both of $g g \rightarrow t h \bar{u}$ and $g g \rightarrow t h \bar{c}$, while in case-(II) only the former process can contribute to $g g \rightarrow t h j$ production. On the other hand, the cross section of $q g \rightarrow t h j$ is almost same in case- $(I)$ and $(I I)$ since it is dominated by the subprocess $u g \rightarrow t h g$.

In the following calculations, we perform the Monte Carlo simulation and explore the sensitivity of $14 \mathrm{TeV}$ LHC to the top-Higgs FCNC couplings through the channel,

$$
p p \rightarrow t\left(\rightarrow b \ell^{+} \nu_{\ell}\right) h(\rightarrow \gamma \gamma) j
$$

which is characterized by two photons appearing as a narrow resonance centered around the Higgs boson mass. So the SM backgrounds to the eq. (3.2) include two parts: the resonant and the non-resonant backgrounds. For the former, they mainly come from the processes that have a Higgs boson decaying to diphoton in the final states, such as Whjj, Zhjj and $t \bar{t} h$ productions. The additional jets in the $W h j j / Z h j j$ events come from the initial or final state radiations. The cross sections of the resonant backgrounds are normalized to their NLO values; for the latter, the main background processes contain the diphoton events produced in association with top quarks, such as $t j \gamma \gamma$ and $t \bar{t} \gamma \gamma$. The $W j j \gamma \gamma$ production can also mimic the signal when one light jet is mistagged as a $b$ jet.

We generate signal and backgrounds events with MadGraph5 and perform the parton shower and the fast detector simulations with PYTHIA [94] and Delphes [95]. When generating the parton level events, we assume $\mu_{R}=\mu_{F}$ to be the default event-by-event value. We cluster the jets by setting the anti- $k_{t}$ algorithm with a cone radius $\Delta R=0.5$ [96]. The $b$-jet tagging efficiency $\left(\epsilon_{b}\right)$ is formulated as a function of the transverse momentum and rapidity of the jets [97]. The mis-tag of QCD jets is assumed to be the default value as in Delphes. In our simulation, we generate 100k events for the signals and backgrounds respectively.

In figure 4 , we show the transverse momentum distributions of two photons in the signal with $\kappa_{t q h}=0.1$ and backgrounds at $14 \mathrm{TeV}$ LHC. Since the two photons in the signal and the resonant backgrounds come from the Higgs boson, they have peaks around $m_{h} / 2$ and possess the harder $p_{T}$ spectrum than those in the non-resonant backgrounds. According to figure 4 , we can impose the cuts $p_{T}^{\gamma_{1}}>50 \mathrm{GeV}$ and $p_{T}^{\gamma_{2}}>25 \mathrm{GeV}$ to suppress the non-resonant backgrounds. 

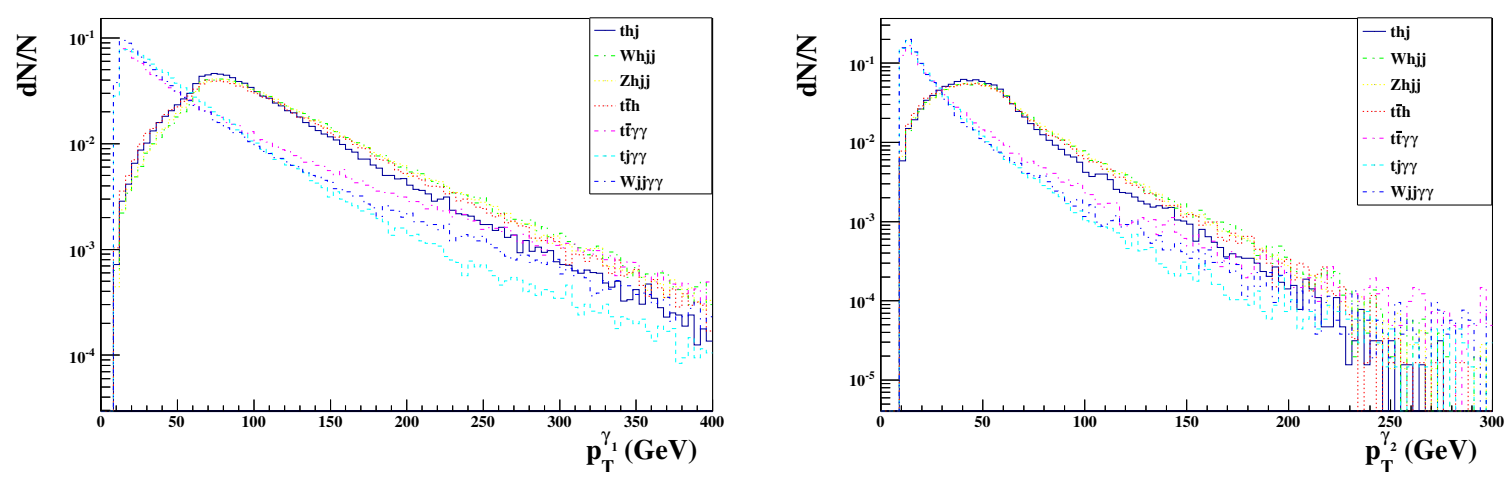

Figure 4. Normalized transverse momentum distributions of two photons in the signals and backgrounds at $14 \mathrm{TeV}$ LHC.

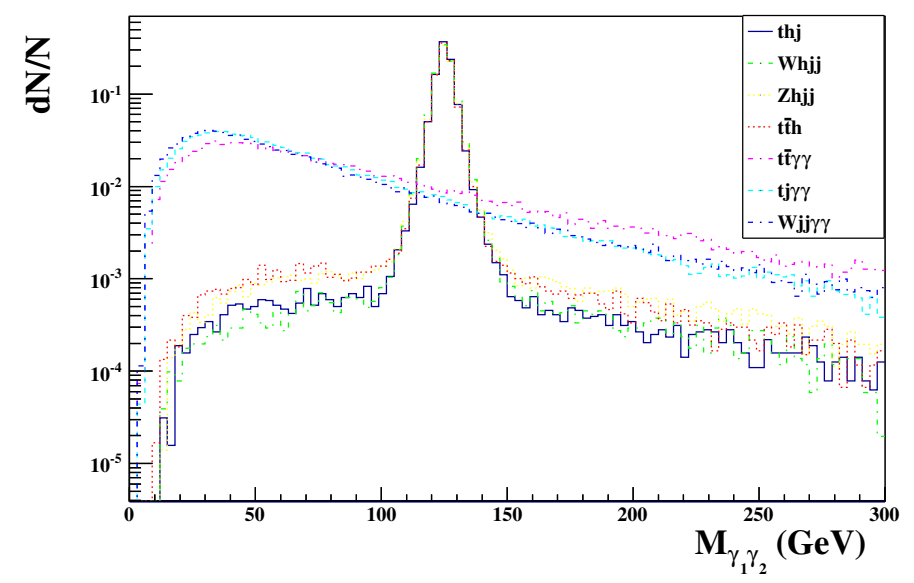

Figure 5. Normalized invariant mass distribution of two photons at $14 \mathrm{TeV}$ LHC.

In figure 5, we present the normalized invariant mass distribution of two photons at $14 \mathrm{TeV}$ LHC. Although the $\gamma \gamma$ decay channel has a small branching ratio, it has the advantage of the good resolution on the $\gamma \gamma$ resonance and is also free from the large QCD backgrounds. From figure 5, we can see that the spreading of the $\gamma \gamma$ invariant-mass peak at $m_{h}$ for the signal and the resonant backgrounds is relatively small. We will use a narrow invariant mass window $\left|M_{\gamma \gamma}-M_{h}\right|<5 \mathrm{GeV}$ to further reduce the non-resonant backgrounds.

In figure 6 , we plot the normalized invariant mass distribution of the $b$ jet and lepton at $14 \mathrm{TeV}$ LHC, which is another effective cut to remove the backgrounds. From figure 6, we can see that the invariant mass $M_{b_{1} \ell_{1}}$ of the signal is always less than the top quark mass since the leading $b$ jet and lepton in our signal come from the same top quark decay. The same feature also appears in the non-resonant background $t j \gamma \gamma$. But other backgrounds can have higher invariant mass $M_{b_{1} \ell_{1}}$ than the signal. Very similar to $M_{b_{1} \ell_{1}}$, the invariant mass distribution of the diphoton and leading light jet $M_{\gamma_{1} \gamma_{2} j}$ also has a peak around the top quark mass in the signal other than the backgrounds, which can be used to further remove the backgrounds. 


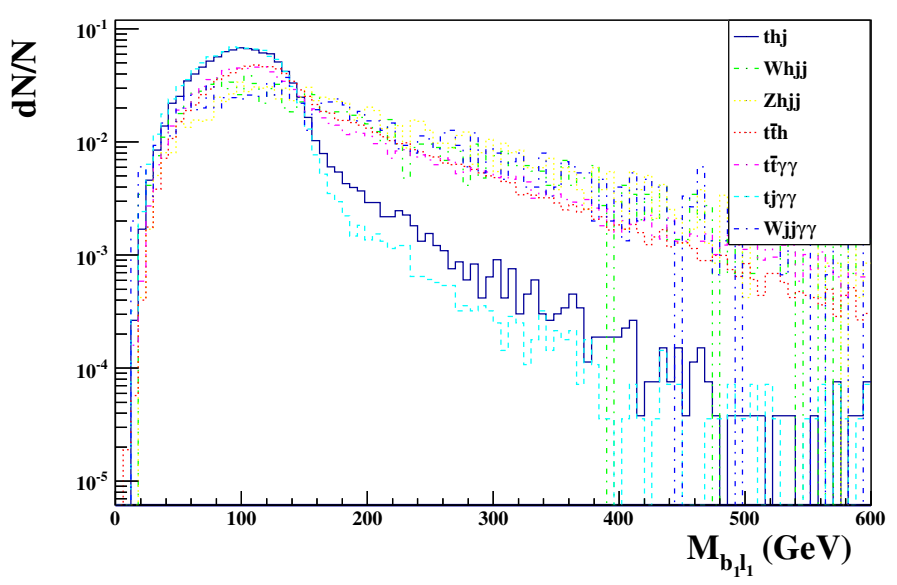

Figure 6. Normalized invariant mass distribution of the $b$ jet and lepton at 14 TeV LHC.

According to the above analysis, events are selected to satisfy the following criteria:

- exact one isolated lepton with $p_{T}\left(\ell_{1}\right)>20 \mathrm{GeV}$ and $\left|\eta_{\ell_{1}}\right|<2$;

- a hard jet with $p_{T}\left(j_{1}\right)>25 \mathrm{GeV}$ and $\left|\eta_{j_{1}}\right|<2.5$ and one $b$-jet with $p_{T}\left(b_{1}\right)>25 \mathrm{GeV}$ and $\left|\eta_{b_{1}}\right|<2.5$

- two photons with $p_{T}^{\gamma_{1}}>50 \mathrm{GeV}$ and $p_{T}^{\gamma_{2}}>25 \mathrm{GeV}$ and their invariant mass $M_{\gamma_{1} \gamma_{2}}$ in the range of $M_{h} \pm 5 \mathrm{GeV}$;

- the invariant mass of $b$-jet and lepton $M_{b \ell}<200 \mathrm{GeV}$;

- the invariant mass of diphoton and leading jet $M_{\gamma_{1} \gamma_{2} j_{1}}<300 \mathrm{GeV}$.

In table 1, we give the cross sections of the signals in the Case- $(I),(I I)$ and $(I I I)$ and backgrounds after the cut flow at $14 \mathrm{TeV}$ LHC, where $\kappa_{t q h}, \kappa_{t u h}$ and $\kappa_{t c h}$ are assumed to be 0.1 respectively. From table 1, we can see that all the non-resonant backgrounds after the cuts of the two photons are reduced by half while the signals and the resonant backgrounds are hurt slightly. Then, we impose the invariant mass cut $M_{b_{1} \ell_{1}}<200 \mathrm{GeV}$ to remove the backgrounds that do not involve the top quark. Since the photon final states have a good energy resolution in the detector, we require that $M_{\gamma_{1} \gamma_{2}}$ be in the range of $120 \mathrm{GeV}<M_{\gamma_{1} \gamma_{2}}<130 \mathrm{GeV}$ and $M_{\gamma_{1} \gamma_{2} j_{1}}<300 \mathrm{GeV}$, which can further suppress the backgrounds by half. So at the end of the cut flow, the largest background is $t j \gamma \gamma$, which is followed by $t \bar{t} h$.

In figure 7 , we plot the contours of statistical significance $S / \sqrt{B}=3 \sigma$ of $p p \rightarrow$ thj at $14 \mathrm{TeV}$ LHC for the Case- $(I),(I I)$ and $(I I I)$ in the plane of $\mathcal{L}-\kappa_{t q h}$. From figure 7 , we can see that the flavor changing couplings $\kappa_{t q h}$ can be probed to $0.047,0.063$ and 0.065 at $3 \sigma$ statistical sensitivity by fully calculating the production of thj for the case $(I),(I I)$ and $(I I I)$ respectively, which correspond to the branching ratios $\operatorname{Br}(t \rightarrow q h)=0.12 \%$, $B r(t \rightarrow u h)=0.23 \%$ and $B r(t \rightarrow c h)=0.26 \%$ at $14 \mathrm{TeV}$ LHC with $\mathcal{L}=3000 \mathrm{fb}^{-1}$. Besides, the corresponding results of the resonant production $p p \rightarrow t \bar{t} \rightarrow t h j$ for each case 

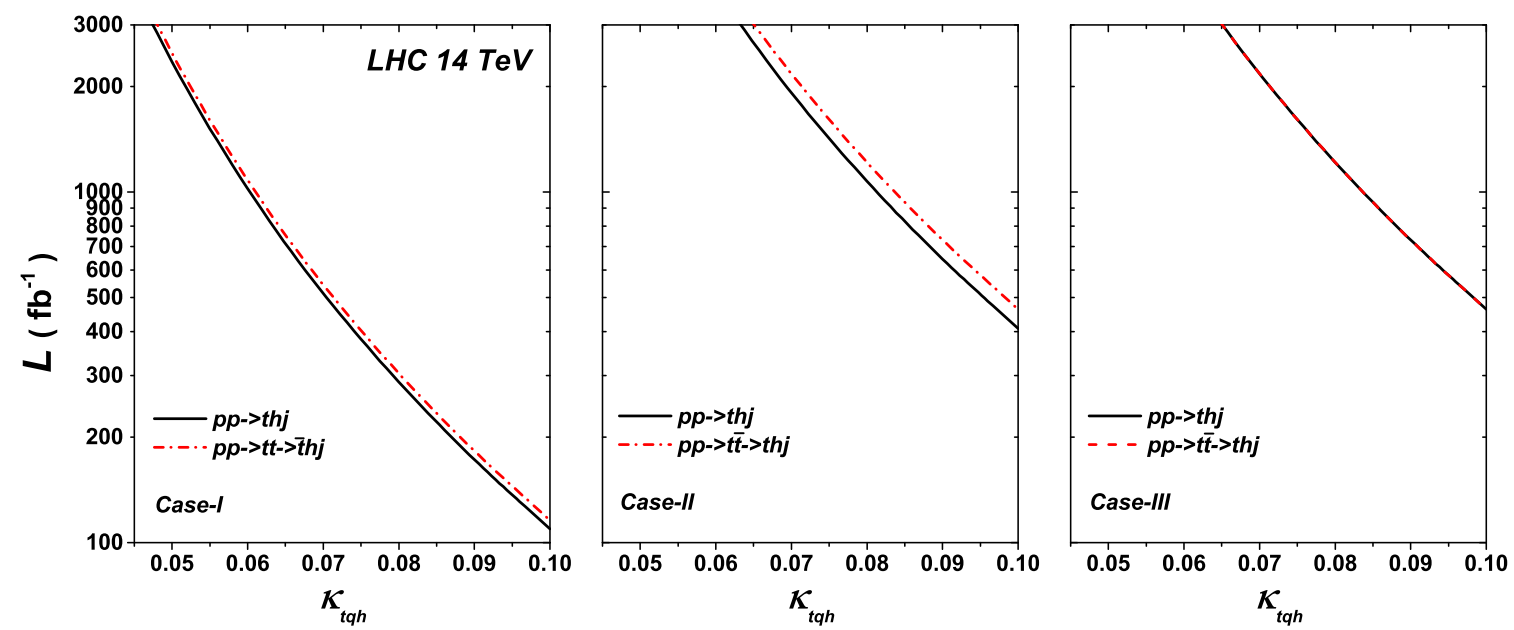

Figure 7. Contour plots in $\mathcal{L}-\kappa_{t q h}$ plane for statistical significance $S / \sqrt{B}=3 \sigma$ of $p p \rightarrow$ thj at $14 \mathrm{TeV}$ LHC. The conjugate processes have been included in the calculations. The cross section of $t \bar{t}$ is normalized to the approximately next-to-next-to-leading order value $\sigma_{t \bar{t}}=920 \mathrm{pb}$ [98]. As a comparison, the corresponding results of the resonant production $p p \rightarrow t \bar{t} \rightarrow t h j$ for each case are also displayed.

\begin{tabular}{|c|c|c|c|c|c|c|c|c|c|}
\hline & \multirow{3}{*}{ Cuts } & \multicolumn{8}{|c|}{ Cross sections $\left(10^{-3} \mathrm{fb}^{-1}\right)$} \\
\hline & & \multicolumn{3}{|c|}{$t h j(t \bar{t})$} & \multirow{2}{*}{$t \bar{t} h$} & \multirow{2}{*}{ Vhjj } & \multirow{2}{*}{$t \bar{t} \gamma \gamma$} & \multirow{2}{*}{$t j \gamma \gamma$} & \multirow{2}{*}{$W j j \gamma \gamma$} \\
\hline & & Case- $I$ & Case- $I I$ & Case- $I I I$ & & & & & \\
\hline (1) & $\begin{array}{cc}\Delta R_{i j}>0.4, & i, j=b, j, \gamma \text { or } \ell \\
p_{T}^{b}>25 \mathrm{GeV}, & \left|\eta_{b}\right|<2.5 \\
p_{T}^{\ell}>20 \mathrm{GeV}, & \left|\eta_{\ell}\right|<2.0 \\
p_{T}^{j}>25 \mathrm{GeV}, & \left|\eta_{j}\right|<2.5\end{array}$ & $2.51(2.32)$ & $1.35(1.16)$ & $1.16(1.16)$ & 0.035 & 0.08 & 4.05 & 2.92 & 2.13 \\
\hline (2) & $p_{T}^{\gamma_{1}}>50 \mathrm{GeV}, p_{T}^{\gamma_{1}}>25 \mathrm{GeV}$ & $2.27(2.10)$ & $1.22(1.05)$ & $1.05(1.05)$ & 0.032 & 0.007 & 1.91 & 1.50 & 1.28 \\
\hline$(3)$ & $M_{b_{1} \ell_{1}}<200 \mathrm{GeV}$ & $2.27(2.10)$ & $1.22(1.05)$ & $1.05(1.05)$ & 0.030 & 0.005 & 1.77 & 1.48 & 0.85 \\
\hline$(4)$ & $\left|M_{\gamma_{1} \gamma_{2}}-m_{h}\right|<5 \mathrm{GeV}$ & $1.99(1.86)$ & $1.06(0.93)$ & $0.93(0.93)$ & 0.022 & 0.004 & 0.07 & 0.09 & - \\
\hline$(5)$ & $M_{\gamma_{1} \gamma_{2} j_{1}}<300 \mathrm{GeV}$ & $1.54(1.46)$ & $0.81(0.73)$ & $0.73(0.73)$ & 0.01 & 0.002 & - & 0.07 & - \\
\hline
\end{tabular}

Table 1. Cut flow of the cross sections for the backgrounds and the signals in the Case- $(I),(I I)$ and (III) at $14 \mathrm{TeV}$ LHC, where $\kappa_{t q h}, \kappa_{t u h}$ and $\kappa_{t c h}$ are assumed to be 0.1 respectively and the symbol "- " stands for the events number less than one. As a comparison, the corresponding results of the resonant production $p p \rightarrow t \bar{t} \rightarrow t h j$ for each case are also listed in the table.

are also displayed. We can see that the LHC sensitivity to the coupling $\kappa_{t q h}$ from the full calculation of $t h j$ production in the Case- $(I I)$ can be improved by about $4 \%$ as a comparison with the resonant production $p p \rightarrow t \bar{t} \rightarrow t h j$, while for other two cases, the enhancement is negligible small. Here it should be mentioned that we normalize the leading order cross section of $t \bar{t}$ to the approximately next-to-next-to-leading order value $\sigma_{t \bar{t}}=920 \mathrm{pb}$ [98]. But the contribution of $q g \rightarrow t h j$ is calculated at the leading order due to lack of the high order correction. So if assuming that the $k$ factor of the process $q g \rightarrow t h j$ be the same as $t \bar{t}$, we can expect the sensitivity to the coupling $\kappa_{t q h}$ from full calculation in Case- $(I)$ and $(I I)$ will be further increased. Compared with other decay modes of the Higgs boson, our result 
is close to that of multi-leptons channel in $t \bar{t} \rightarrow t h\left(\rightarrow W W^{*}, \tau^{+} \tau^{-}, Z Z^{*}\right) j$ production [99], based native scaling in cross section and luminosity at $14 \mathrm{TeV}$ LHC. Although the decay of $h \rightarrow b \bar{b}$ has a larger branching ratio and seems more promising [73], the analysis was performed at the parton level without including the parton shower and detector effects. However, these effects are important for the Higgs mass reconstruction and can severely reduce the cut efficiency of Higgs mass window in $b \bar{b}$ channel.

\section{Conclusion}

In the work, we investigated the process $p p \rightarrow t h j$ induced by the top-Higgs FCNC couplings at the LHC. We found that the cross section of $p p \rightarrow$ thj can be sizably enhanced in contrast with the SM predictions at 8 and $14 \mathrm{TeV}$ LHC under the current constraints. We studied the observability of top-Higgs FCNC couplings through the process $p p \rightarrow t\left(\rightarrow b \ell^{+} \nu_{\ell}\right) h(\rightarrow \gamma \gamma) j$ by including the resonant and non-resonant $h j$ production at $14 \mathrm{TeV}$ LHC. Compared with the resonant production $p p \rightarrow t \bar{t} \rightarrow t h j$, such a full calculation can increase the LHC $3 \sigma$ sensitivity to $B r(t \rightarrow q h)$ by $4 \%$ and $B r(t \rightarrow u h)$ by $10 \%$ at $14 \mathrm{TeV}$ LHC with $\mathcal{L}=3000 \mathrm{fb}^{-1}$ because of the contribution of the non-resonant production $q g \rightarrow t h j$. Finally, the branching ratios $B r(t \rightarrow q h), B r(t \rightarrow u h)$ and $B r(t \rightarrow c h)$ can be respectively probed to $0.12 \%, 0.23 \%$ and $0.26 \%$ at $3 \sigma$ level at $14 \mathrm{TeV}$ LHC with $\mathcal{L}=3000 \mathrm{fb}^{-1}$.

\section{Acknowledgments}

This work was supported by the Australian Research Council, the National Natural Science Foundation of China (NNSFC) under grants Nos. 11405047, 11275057, 11305049 and 11405047, by Specialized Research Fund for the Doctoral Program of Higher Education under Grant No. 20134104120002.

Open Access. This article is distributed under the terms of the Creative Commons Attribution License (CC-BY 4.0), which permits any use, distribution and reproduction in any medium, provided the original author(s) and source are credited.

\section{References}

[1] ATLAS collaboration, Combined search for the Standard Model Higgs boson using up to $4.9 \mathrm{fb}^{-1}$ of pp collision data at $\sqrt{s}=7 \mathrm{TeV}$ with the ATLAS detector at the LHC, Phys. Lett. B 710 (2012) 49 [arXiv:1202.1408] [INSPIRE].

[2] CMS collaboration, Combined results of searches for the standard model Higgs boson in pp collisions at $\sqrt{s}=7 \mathrm{TeV}$, Phys. Lett. B 710 (2012) 26 [arXiv:1202.1488] [INSPIRE].

[3] S. Dawson et al., Working Group Report: Higgs Boson, arXiv:1310.8361 [INSPIRE].

[4] C. Englert et al., Precision Measurements of Higgs Couplings: Implications for New Physics Scales, J. Phys. G 41 (2014) 113001 [arXiv:1403.7191] [InSPIRE].

[5] C.-S. Li, H.-T. Li and D.-Y. Shao, Some recent theoretical progress in Higgs boson and top quark physics at hadron colliders, Chin. Sci. Bull. 59 (2014) 3709 [arXiv:1401.1101] [INSPIRE]. 
[6] Top Quark Working Group, K. Agashe et al., Working Group Report: Top Quark, arXiv:1311.2028 [INSPIRE].

[7] C. Zhang and S. Willenbrock, Effective Field Theory for Top Quark Physics, Nuovo Cim. C 033 (2010) 285 [arXiv: 1008.3155] [INSPIRE].

[8] W. Bernreuther, Top quark physics at the LHC, J. Phys. G 35 (2008) 083001 [arXiv: 0805.1333] [INSPIRE].

[9] J.A. Aguilar-Saavedra, A Minimal set of top anomalous couplings, Nucl. Phys. B 812 (2009) 181 [arXiv: 0811.3842] [INSPIRE].

[10] J.A. Aguilar-Saavedra, A Minimal set of top-Higgs anomalous couplings, Nucl. Phys. B 821 (2009) 215 [arXiv:0904.2387] [INSPIRE].

[11] E.W.N. Glover et al., Top quark physics at colliders, Acta Phys. Polon. B 35 (2004) 2671 [hep-ph/0410110] [INSPIRE].

[12] D. Chakraborty, J. Konigsberg and D.L. Rainwater, Review of top quark physics, Ann. Rev. Nucl. Part. Sci. 53 (2003) 301 [hep-ph/0303092] [INSPIRE].

[13] M. Beneke et al., Top quark physics, hep-ph/0003033 [INSPIRE].

[14] T. Han, The 'Top Priority' at the LHC, Int. J. Mod. Phys. A 23 (2008) 4107 [arXiv: 0804.3178] [INSPIRE].

[15] G. Eilam, J.L. Hewett and A. Soni, Rare decays of the top quark in the standard and two Higgs doublet models, Phys. Rev. D 44 (1991) 1473 [Erratum ibid. D 59 (1999) 039901] [INSPIRE].

[16] B. Mele, S. Petrarca and A. Soddu, A New evaluation of the $t \rightarrow c H$ decay width in the standard model, Phys. Lett. B 435 (1998) 401 [hep-ph/9805498] [INSPIRE].

[17] A. Cordero-Cid, J.M. Hernandez, G. Tavares-Velasco and J.J. Toscano, Rare top quark decay $t \rightarrow u_{1} \bar{u}_{2} u_{2}$ in the standard model, Phys. Rev. D 73 (2006) 094005 [hep-ph/0411188] [INSPIRE].

[18] G. Eilam, M. Frank and I. Turan, Rare decay of the top $t \rightarrow$ cgg in the standard model, Phys. Rev. D 73 (2006) 053011 [hep-ph/0601151] [INSPIRE].

[19] J.J. Cao, C. Han, L. Wu, J.M. Yang and M. Zhang, SUSY induced top quark FCNC decay $t \rightarrow c h$ after Run I of LHC, Eur. Phys. J. C 74 (2014) 3058 [arXiv:1404.1241] [InSPIRE].

[20] T.-J. Gao, T.-F. Feng, F. Sun, H.-B. Zhang and S.-M. Zhao, Top quark decay to a $125 \mathrm{GeV}$ Higgs in BLMSSM, arXiv:1404.3289 [INSPIRE].

[21] S. Bejar, J. Guasch, D. Lopez-Val and J. Solà, FCNC-induced heavy-quark events at the LHC from Supersymmetry, Phys. Lett. B 668 (2008) 364 [arXiv:0805.0973] [INSPIRE].

[22] J.J. Cao et al., SUSY-induced FCNC top-quark processes at the large hadron collider, Phys. Rev. D 75 (2007) 075021 [hep-ph/0702264] [INSPIRE].

[23] M. Frank and I. Turan, Rare decay of the top $t \rightarrow c l \bar{l}$ and single top production at ILC, Phys. Rev. D 74 (2006) 073014 [hep-ph/0609069] [INSPIRE].

[24] S. Bejar, J. Guasch and J. Solà, Production and FCNC decay of supersymmetric Higgs bosons into heavy quarks in the LHC, JHEP 10 (2005) 113 [hep-ph/0508043] [INSPIRE].

[25] J.L. Diaz-Cruz, H.-J. He and C.P. Yuan, Soft SUSY breaking, stop scharm mixing and Higgs signatures, Phys. Lett. B 530 (2002) 179 [hep-ph/0103178] [INSPIRE].

[26] J. Guasch and J. Solà, FCNC top quark decays: A Door to SUSY physics in high luminosity colliders?, Nucl. Phys. B 562 (1999) 3 [hep-ph/9906268] [InSPIRE]. 
[27] C.-S. Li, R.J. Oakes and J.M. Yang, Rare decay of the top quark in the minimal supersymmetric model, Phys. Rev. D 49 (1994) 293 [Erratum ibid. D 56 (1997) 3156] [INSPIRE].

[28] J.-M. Yang and C.-S. Li, Top quark rare decay $t \rightarrow c H^{i}$ in the minimal supersymmetric model, Phys. Rev. D 49 (1994) 3412 [Erratum ibid. D 51 (1995) 3974] [INSPIRE].

[29] Z.-x. Heng, G.-r. Lu, L. Wu and J.M. Yang, Top quark three-body decays in R-violating MSSM, Phys. Rev. D 79 (2009) 094029 [arXiv:0904.0597] [INSPIRE].

[30] J.J. Cao, Z.-x. Heng, L. Wu and J.M. Yang, R-parity violating effects in top quark FCNC productions at LHC, Phys. Rev. D 79 (2009) 054003 [arXiv: 0812.1698] [INSPIRE].

[31] J.M. Yang, B.-L. Young and X. Zhang, Flavor changing top quark decays in R-parity violating SUSY, Phys. Rev. D 58 (1998) 055001 [hep-ph/9705341] [INSPIRE].

[32] G. Eilam, A. Gemintern, T. Han, J.M. Yang and X. Zhang, Top quark rare decay $t \rightarrow$ ch in R-parity violating SUSY, Phys. Lett. B 510 (2001) 227 [hep-ph/0102037] [INSPIRE].

[33] T. Han and R. Ruiz, Higgs Bosons from Top Quark Decays, Phys. Rev. D 89 (2014) 074045 [arXiv: 1312.3324] [INSPIRE].

[34] K.-F. Chen, W.-S. Hou, C. Kao and M. Kohda, When the Higgs meets the Top: Search for $t \rightarrow c h^{0}$ at the LHC, Phys. Lett. B 725 (2013) 378 [arXiv:1304.8037] [INSPIRE].

[35] C. Kao, H.-Y. Cheng, W.-S. Hou and J. Sayre, Top Decays with Flavor Changing Neutral Higgs Interactions at the LHC, Phys. Lett. B 716 (2012) 225 [arXiv:1112.1707] [INSPIRE].

[36] A. Arhrib, K. Cheung, C.-W. Chiang and T.-C. Yuan, Single top-quark production in flavor-changing Z' models, Phys. Rev. D 73 (2006) 075015 [hep-ph/0602175] [INSPIRE].

[37] M. Frank and I. Turan, $t \rightarrow c g, c \gamma, c Z$ in the left-right supersymmetric model, Phys. Rev. D 72 (2005) 035008 [hep-ph/0506197] [INSPIRE].

[38] J.A. Aguilar-Saavedra and B.M. Nobre, Rare top decays $t \rightarrow c \gamma, t \rightarrow c g$ and CKM unitarity, Phys. Lett. B 553 (2003) 251 [hep-ph/0210360] [INSPIRE].

[39] E.O. Iltan and I. Turan, The Flavor changing $\vec{t} c l_{1}^{-} l_{2}^{+}$decay in the general two Higgs doublet model, Phys. Rev. D 67 (2003) 015004 [hep-ph/0207087] [InSPIRE].

[40] S. Bejar, J. Guasch and J. Solà, Loop induced flavor changing neutral decays of the top quark in a general two Higgs doublet model, Nucl. Phys. B 600 (2001) 21 [hep-ph/0011091] [INSPIRE].

[41] F. del Aguila, J.A. Aguilar-Saavedra and R. Miquel, Constraints on top couplings in models with exotic quarks, Phys. Rev. Lett. 82 (1999) 1628 [hep-ph/9808400] [INSPIRE].

[42] S. Bar-Shalom, G. Eilam, A. Soni and J. Wudka, Probing the flavor changing tc vertex via tree level processes: $e^{+} e^{-} \rightarrow t \bar{c} \nu_{e} \bar{\nu}_{e}, t \bar{c} e^{+} e^{-}$, and $t \rightarrow c W^{+} W^{-}$, Phys. Rev. Lett. 79 (1997) 1217 [hep-ph/9703221] [INSPIRE].

[43] W.-S. Hou, G.-L. Lin and C.-Y. Ma, Flavor changing neutral Higgs couplings and top charm production at next linear collider, Phys. Rev. D 56 (1997) 7434 [hep-ph/9708228] [INSPIRE].

[44] J.L. Diaz-Cruz, R. Martinez, M.A. Perez and A. Rosado, Flavor Changing Radiative Decay of The $t$ Quark, Phys. Rev. D 41 (1990) 891 [InSPIRE].

[45] D. Atwood, L. Reina and A. Soni, Phenomenology of two Higgs doublet models with flavor changing neutral currents, Phys. Rev. D 55 (1997) 3156 [hep-ph/9609279] [INSPIRE].

[46] B. Yang, N. Liu and J. Han, Top Quark FCNC Decay to 125 GeV Higgs boson in the Littlest Higgs Model with T-parity, arXiv:1308.4852 [INSPIRE]. 
[47] L. Wang, L. Wu and J.M. Yang, Top quark forward-backward asymmetry and charge asymmetry in left-right twin Higgs model, Phys. Rev. D 85 (2012) 075017 [arXiv:1111.4771] [INSPIRE].

[48] J.J. Cao, K. Hikasa, L. Wang, L. Wu and J.M. Yang, Testing new physics models by top charge asymmetry and polarization at the LHC, Phys. Rev. D 85 (2012) 014025 [arXiv: 1109.6543] [INSPIRE].

[49] G. Liu and H.-j. Zhang, Single top production associated with a neutral scalar at LHC in topcolor-assisted technicolor, Chin. Phys. C 32 (2008) 697 [arXiv:0708.1553] [INSPIRE].

[50] M. Gorbahn and U. Haisch, Searching for $t \rightarrow c(u) h$ with dipole moments, JHEP 06 (2014) 033 [arXiv: 1404.4873] [INSPIRE].

[51] C.-X. Yue, S.-Y. Cao and Q.-G. Zeng, Light axigluon and single top production at the LHC, JHEP 04 (2014) 170 [arXiv: 1401.5159] [INSPIRE].

[52] C. Han, N. Liu, L. Wu and J.M. Yang, Probing topcolor-assisted technicolor from top charge asymmetry and triple-top production at the LHC, Phys. Lett. B 714 (2012) 295 [arXiv:1203.2321] [INSPIRE].

[53] J.J. Cao, L. Wu and J.M. Yang, New physics effects on top quark spin correlation and polarization at the LHC: a comparative study in different models, Phys. Rev. D 83 (2011) 034024 [arXiv: 1011.5564] [INSPIRE].

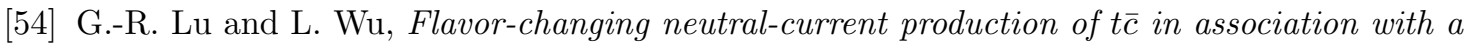
neutral top-Higgs at LHC in the topcolor-assisted technicolor model, Chin. Phys. Lett. 27 (2010) 031401 [INSPIRE].

[55] J. Drobnak, S. Fajfer and J.F. Kamenik, Signatures of NP models in top FCNC decay $t \rightarrow c(u) \ell^{+} \ell^{-}$, JHEP 03 (2009) 077 [arXiv: 0812.0294] [INSPIRE].

[56] J.J. Cao, Z.-h. Xiong and J.M. Yang, SUSY induced top quark FCNC processes at linear colliders, Nucl. Phys. B 651 (2003) 87 [hep-ph/0208035] [INSPIRE].

[57] J.J. Cao, L. Wang, L. Wu and J.M. Yang, Top quark forward-backward asymmetry, FCNC decays and like-sign pair production as a joint probe of new physics, Phys. Rev. D 84 (2011) 074001 [arXiv: 1101.4456 ] [INSPIRE].

[58] J.J. Cao, Z.-x. Heng, L. Wu and J.M. Yang, Top quark forward-backward asymmetry at the Tevatron: A Comparative study in different new physics models, Phys. Rev. D 81 (2010) 014016 [arXiv: 0912.1447] [inSPIRE].

[59] A. Azatov, M. Toharia and L. Zhu, Higgs Mediated FCNC's in Warped Extra Dimensions, Phys. Rev. D 80 (2009) 035016 [arXiv:0906.1990] [INSPIRE].

[60] K. Agashe and R. Contino, Composite Higgs-Mediated FCNC, Phys. Rev. D 80 (2009) 075016 [arXiv: 0906.1542] [INSPIRE].

[61] P.M. Ferreira, R.B. Guedes and R. Santos, Combined effects of strong and electroweak FCNC effective operators in top quark physics at the CERN LHC, Phys. Rev. D 77 (2008) 114008 [arXiv: 0802.2075] [INSPIRE].

[62] F. Larios, R. Martinez and M.A. Perez, New physics effects in the flavor-changing neutral couplings of the top quark, Int. J. Mod. Phys. A 21 (2006) 3473 [hep-ph/0605003] [INSPIRE].

[63] J.M. Yang, Probing new physics from top quark FCNC processes at linear colliders: A Mini review, Annals Phys. 316 (2005) 529 [hep-ph/0409351] [INSPIRE].

[64] J.A. Aguilar-Saavedra, Top flavor-changing neutral interactions: Theoretical expectations and experimental detection, Acta Phys. Polon. B 35 (2004) 2695 [hep-ph/0409342] [INSPIRE]. 
[65] ATLAS, CDF, CMS, D0 collaborations, E. Yazgan, Flavor changing neutral currents in top quark production and decay, arXiv:1312.5435 [INSPIRE].

[66] ATLAS collaboration, Search for top quark decays $t \rightarrow q H$ with $H \rightarrow \gamma \gamma$ using the ATLAS detector, JHEP 06 (2014) 008 [arXiv: 1403.6293] [INSPIRE].

[67] CMS Collaboration, Combined multilepton and diphoton limit on t to $\mathrm{cH}$, CMS-PAS-HIG-13-034 (2014) [INSPIRE].

[68] A. Greljo, J.F. Kamenik and J. Kopp, Disentangling Flavor Violation in the Top-Higgs Sector at the LHC, JHEP 07 (2014) 046 [arXiv:1404.1278] [INSPIRE].

[69] S. Khatibi and M.M. Najafabadi, Probing the Anomalous FCNC Interactions in Top-Higgs Final State and Charge Ratio Approach, Phys. Rev. D 89 (2014) 054011 [arXiv:1402.3073] [INSPIRE].

[70] D. Atwood, S.K. Gupta and A. Soni, Constraining the flavor changing Higgs couplings to the top-quark at the LHC, JHEP 10 (2014) 057 [arXiv:1305.2427] [INSPIRE].

[71] Y. Wang, F.P. Huang, C.-S. Li, B.H. Li, D.-Y. Shao and J. Wang, Constraints on flavor-changing neutral-current Htq couplings from the signal of $t H$ associated production with QCD next-to-leading order accuracy at the LHC, Phys. Rev. D 86 (2012) 094014 [arXiv:1208.2902] [INSPIRE].

[72] D. Lopez-Val, J. Guasch and J. Solà, Single top-quark production by strong and electroweak supersymmetric flavor-changing interactions at the LHC, JHEP 12 (2007) 054 [arXiv: 0710.0587] [INSPIRE].

[73] J.A. Aguilar-Saavedra and G.C. Branco, Probing top flavor changing neutral scalar couplings at the CERN LHC, Phys. Lett. B 495 (2000) 347 [hep-ph/0004190] [INSPIRE].

[74] T.M.P. Tait and C.-P. Yuan, Single top quark production as a window to physics beyond the standard model, Phys. Rev. D 63 (2000) 014018 [hep-ph/0007298] [INSPIRE].

[75] F. Maltoni, K. Paul, T. Stelzer and S. Willenbrock, Associated production of Higgs and single top at hadron colliders, Phys. Rev. D 64 (2001) 094023 [hep-ph/0106293] [INSPIRE].

[76] M. Farina, C. Grojean, F. Maltoni, E. Salvioni and A. Thamm, Lifting degeneracies in Higgs couplings using single top production in association with a Higgs boson, JHEP 05 (2013) 022 [arXiv: 1211.3736] [INSPIRE].

[77] S. Biswas, E. Gabrielli and B. Mele, Single top and Higgs associated production as a probe of

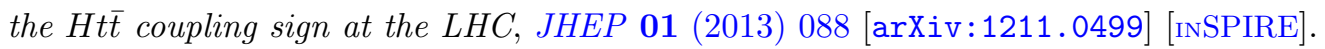

[78] J.R. Ellis, D.S. Hwang, K. Sakurai and M. Takeuchi, Disentangling Higgs-Top Couplings in Associated Production, JHEP 04 (2014) 004 [arXiv: 1312.5736] [INSPIRE].

[79] C. Englert and E. Re, Bounding the top Yukawa coupling with Higgs-associated single-top production, Phys. Rev. D 89 (2014) 073020 [arXiv:1402.0445] [INSPIRE].

[80] J. Chang, K. Cheung, J.S. Lee and C.-T. Lu, Probing the Top-Yukawa Coupling in Associated Higgs production with a Single Top Quark, JHEP 05 (2014) 062 [arXiv: 1403.2053] [INSPIRE].

[81] A. Kobakhidze, L. Wu and J. Yue, Anomalous Top-Higgs Couplings and Top Polarisation in Single Top and Higgs Associated Production at the LHC, JHEP 10 (2014) 100 [arXiv: 1406.1961] [INSPIRE].

[82] C.-S. Li, R.J. Oakes and T.C. Yuan, QCD corrections to $t \rightarrow W^{+}+b$, Phys. Rev. D 43 (1991) 3759 [INSPIRE]. 
[83] C. Zhang and F. Maltoni, Top-quark decay into Higgs boson and a light quark at next-to-leading order in QCD, Phys. Rev. D 88 (2013) 054005 [arXiv: 1305.7386] [INSPIRE].

[84] J. Drobnak, S. Fajfer and J.F. Kamenik, Flavor Changing Neutral Coupling Mediated Radiative Top Quark Decays at Next-to-Leading Order in QCD, Phys. Rev. Lett. 104 (2010) 252001 [arXiv: 1004.0620] [INSPIRE].

[85] J.J. Zhang et al., Next-to-leading order QCD corrections to the top quark decay via model-independent FCNC couplings, Phys. Rev. Lett. 102 (2009) 072001 [arXiv:0810. 3889] [INSPIRE].

[86] J.J. Cao, G. Eilam, K.-i. Hikasa and J.M. Yang, Experimental constraints on stop-scharm flavor mixing and implications in top-quark FCNC processes, Phys. Rev. D 74 (2006) 031701 [hep-ph/0604163] [INSPIRE].

[87] G.C. Branco, W. Grimus and L. Lavoura, Relating the scalar flavor changing neutral couplings to the CKM matrix, Phys. Lett. B 380 (1996) 119 [hep-ph/9601383] [INSPIRE].

[88] A.S. Joshipura and S.D. Rindani, Naturally suppressed flavor violations in two Higgs doublet models, Phys. Lett. B 260 (1991) 149 [INSPIRE].

[89] T.P. Cheng and M. Sher, Mass Matrix Ansatz and Flavor Nonconservation in Models with Multiple Higgs Doublets, Phys. Rev. D 35 (1987) 3484 [InSPIRE].

[90] A. Alloul, N.D. Christensen, C. Degrande, C. Duhr and B. Fuks, FeynRules 2.0 - A complete toolbox for tree-level phenomenology, Comput. Phys. Commun. 185 (2014) 2250 [arXiv:1310.1921] [INSPIRE].

[91] J. Alwall, M. Herquet, F. Maltoni, O. Mattelaer and T. Stelzer, MadGraph 5: Going Beyond, JHEP 06 (2011) 128 [arXiv:1106.0522] [INSPIRE].

[92] J. Pumplin, A. Belyaev, J. Huston, D. Stump and W.K. Tung, Parton distributions and the strong coupling: CTEQ6AB PDFs, JHEP 02 (2006) 032 [hep-ph/0512167] [INSPIRE].

[93] Particle Data Group collaboration, J. Beringer et al., Review of Particle Physics (RPP), Phys. Rev. D 86 (2012) 010001 [INSPIRE].

[94] T. Sjöstrand, S. Mrenna and P.Z. Skands, PYTHIA 6.4 Physics and Manual, JHEP 05 (2006) 026 [hep-ph/0603175] [INSPIRE].

[95] DELPHES 3 collaboration, J. de Favereau et al., DELPHES 3, A modular framework for fast simulation of a generic collider experiment, JHEP 02 (2014) 057 [arXiv:1307.6346] [INSPIRE].

[96] M. Cacciari, G.P. Salam and G. Soyez, The Anti-k $k_{t}$ jet clustering algorithm, JHEP 04 (2008) 063 [arXiv: 0802.1189] [INSPIRE].

[97] CMS Collaboration, b-Jet Identification in the CMS Experiment, CMS-PAS-BTV-11-004 (2012) [INSPIRE].

[98] N. Kidonakis, The top quark rapidity distribution and forward-backward asymmetry, Phys. Rev. D 84 (2011) 011504 [arXiv: 1105.5167] [INSPIRE].

[99] N. Craig et al., Searching for $t \rightarrow$ ch with Multi-Leptons, Phys. Rev. D 86 (2012) 075002 [arXiv: 1207.6794] [INSPIRE]. 